\title{
Mechanism of Action of Novel Pyrazole Carboxamide Containing a Diarylamine Scaffold against Rhizoctonia solani
}

Yongtian Zhao ${ }^{1,2}$, Na Yang ${ }^{1}$, Yiming Deng ${ }^{1}, \mathrm{Ke} \mathrm{Tao}^{1}$, Hong Jin ${ }^{1, *}$, and Taiping Hou ${ }^{1}$

1. Key Laboratory of Bio-Resource and Eco-Environment of Ministry of Education, College of Life Sciences, Sichuan University, Chengdu, Sichuan 610065, China.

2. College of Agroforestry and Health, Sichuan Radio and TV University, Chengdu, Sichuan 610073, China.

*E-mail address: jinhong@scu.edu.cn (H. Jin) 


\section{Content}

\section{Experimental methods}

2. Table S1. The sequences of primers and internal reference gene of actin.

3. Table S2. GO notes in cell component (CC), molecular function (MF) and biological process (BP).

4. Table S3. Related pathways involve differential expression enzymes and genes in mitochondria. 


\section{MATERIALS AND METHODS}

Antifungal Assay. The antifungal assay of compound SCU2028 against $R$. solani was determined by mycelial growth rate method. Compound SCU2028 was diluted into different concentration gradient drugs with acetone as the treatment group, and an equal volume of acetone was used as the blank control, the acetone solution of thifluzamide was used as a positive control, each concentration was repeated three times. The calculation of the virulence equation is based on the curve drawn by the inhibition rate and the logarithm of the concentration, the inhibition rate of mycelium growth was calculated according to the following formula:

$$
\text { Inhibition Rate }(\%)=\frac{\mathrm{Cd}(\mathrm{cm})-\mathrm{Td}(\mathrm{cm})}{\mathrm{Cd}(\mathrm{cm})-0.7(\mathrm{~cm})} \times 100
$$

Where $\mathrm{Cd}(\mathrm{cm})$ is the average colony diameter of the control group, $\mathrm{Td}(\mathrm{cm})$ is the average colony diameter of the treatment group.

Observation of the Mycelium Morphology and Ultrastructure. Morphological Observation by Scanning Electron Microscopy (SEM). Concentrations of compound SCU2028 dissolved in acetone were its $\mathrm{EC}_{50}$ and $\mathrm{EC}_{90}$ values. The control group was added with the same volume of sterile acetone. Taked out the mycelium, $2.5 \%$ glutaraldehyde was fixed at $4{ }^{\circ} \mathrm{C}$ for $4-6 \mathrm{~h}$, the mycelium was washed with PBS buffer for 2-3 times, then dehydrated with ethanol gradient $(30 \%, 50 \%, 70 \%, 85 \%$, $90 \%$ once respectively, $100 \%$ twice, 20 min each time), the dehydrated mycelium was dried in vacuum, finally gold plating was carried out. The mycelia were observed and photographed on JSM-7500F (JEOL, Japan) scanning electron microscope.

Ultrastructural Observation by Transmission Electron Microscopy (TEM). Concentrations of compound SCU2028 dissolved in acetone were its $\mathrm{EC}_{50}$ and $\mathrm{EC}_{90}$ values. The control group was added with the same volume of sterile acetone. The mycelium was put into $2.5 \%$ glutaraldehyde at $4{ }^{\circ} \mathrm{C}$ for $4-6 \mathrm{~h}$, poured out the fixing 
solution, washed it with PBS buffer for 2-3 times, fixed it with $1 \%$ osmium acid for 1-2 h, rinsed it with PBS buffer again for 2-3 times, then dehydrated it with gradient concentration $(50 \%, 70 \%, 80 \%, 90 \%, 95 \%)$ ethanol for $15 \mathrm{~min}$, and then treated it with $100 \%$ ethanol for $20 \mathrm{~min}$. The mixture of embedment agent and acetone (1:1) was treated for $1 \mathrm{~h}$, then the mixture of embedment agent and acetone $(2: 1)$ was permeated for $3 \mathrm{~h}$, and the samples were transferred to a new drying tube for overnight penetration of pure embedment agent. The samples were transferred to a new drying tube again, heated and polymerized overnight at $70{ }^{\circ} \mathrm{C}$ with embedding agent. The polymerized samples were sliced into ultra-thin sections by a microtome. After double staining with uranyl acetate and lead citrate, the mycelia were observed and photographed on JEM-1230 (Tokyo, Japan) transmission electron microscope.

Detection of the Mitochondrial Membrane Potential (MMP). The concentration of SCU2028 was $\mathrm{EC}_{90}$, an equal volume of acetone was added as the control. After treated with SCU2028 for $24 \mathrm{~h}$, the mycelia stained with $10 \mu \mathrm{g} / \mathrm{ml} \mathrm{Rh} 123$, then incubated in the dark for $30 \mathrm{~min}$ at $28^{\circ} \mathrm{C}$. After incubation, the mycelia were washed 2-3 times with PBS buffer, and then the samples were observed and photographed by A1R MP+ (Nikon, Japan) confocal laser scanning microscope. The excitation wavelength was $488 \mathrm{~nm}$, and the emission wavelength was $515 \mathrm{~nm}$.

Label Free Quantitative Proteomics Analysis (LFQPA). Mycelium treatment. Concentration of compound SCU2028 dissolved in acetone was its $\mathrm{EC}_{90}$ value for 24 h. The control group was added with the same volume of sterile acetone. Each treatment group was repeated three times.

Protein Extraction and Trypsin Digest. The samples were ground into powder by liquid nitrogen and then added with pyrolysis solution (8M urea; 30mM HEPES; 1mM PMSF; 2mM EDTA; 10mM DTT) for $5 \mathrm{~min}$. Then centrifugation at 20,000 $\mathrm{g}$ 
for $30 \mathrm{~min}$ and took the supernatant. DTT was added to the final concentration of 10 $\mathrm{mM}$, and watered bath at $56^{\circ} \mathrm{C}$ for $1 \mathrm{~h}$. After removal, IAM was rapidly added to the final concentration of $55 \mathrm{mM}$, and the darkroom was stationary for $1 \mathrm{~h}$. Bradford method was used to quantify the protein, and SDS-PAGE was used to detect the quality of total protein extraction.

$L C-M S / M S$ Analysis. Each sample was taken $20 \mu \mathrm{g}$ and added to $10 \mathrm{~K}$ ultrafiltration tube. The waste liquid was discarded after centrifugation at $14000 \mathrm{~g}$ for $40 \mathrm{~min}$ at $4{ }^{\circ} \mathrm{C}$. Added $200 \mathrm{ml} 25 \mathrm{mM}$ ammonium bicarbonate, $14000 \mathrm{~g}$, centrifuge at $4{ }^{\circ} \mathrm{C}$ for $40 \mathrm{~min}$, discarded the waste liquid. Repeated the steps twice. $0.9 \mu \mathrm{g}$ Trypsin was added and bathed at $37{ }^{\circ} \mathrm{C}$ for $24 \mathrm{~h}$. The lyophilized enzymatic hydrolysate was then used to dissolve the peptide fragments with $0.1 \% \mathrm{FA}$ and packaged into $40 \mathrm{ml}$ tubes for mass spectrometric analysis. The samples for mass spectrometry analysis were separated by Dionex Ultimate 3000 nano LC system liquid phase system (Thermo Scientific, USA). The mobile phase A contains water and $0.1 \%$ FA, while the mobile phase B contains acetonitrile and $0.1 \%$ FA. The flow rate was maintained at 400 nl/min. Peptide segments were separated by liquid phase system and ionized by implantation ion source. The signal of peptide segments was detected by Q-Exactive mass spectrometer. The eluates were directly entered Q-Exactive MS (Thermo Fisher Scientific, USA), setting in positive ion mode and data-dependent manner with full MS scan from 350-2000 m/z, full scan resolution at 70,000, MS/MS scan resoltion at 17,500 .

Molecular Docking. Molecular docking studies were performed to investigate the binding mode of the compound to the cytochrome c oxidase using Autodock vina 1.1.2. The three-dimensional (3D) structure of the cytochrome oxidase (PDB ID: 1V54) was downloaded from RCSB Protein Data Bank (www.rcsb.org). The 3D 
structure of 2028 was drawn by ChemBioDraw Ultra 14.0 and ChemBio3D Ultra 14.0 softwares. The AutoDock Tools 1.5.6 package was employed to generate the docking input files. The ligand was prepared for docking by merging non-polar hydrogen atoms and defining rotatable bonds. The search grid of cytochrome oxidase was identified as center_x: 76.075, center_y: 286.366, and center_z: 209.317 with dimensions size_x: 15, size_y: 15, and size_z: 15 . The value of exhaustiveness was set to 20. For Vina docking, the default parameters were used if it was not mentioned. The best-scoring pose as judged by the Vina docking score was chosen and visually analyzed using PyMoL 1.7.6 software (www.pymol.org). 
2. Table S1. The sequences of primers and internal reference gene of actin.

\begin{tabular}{cc}
\hline Gene & Primer sequences (5' to 3') \\
Sdh2-F & CCTCAAGAACGACAACCC \\
Sdh2-R & GCGAGCATACAGCCAGAA \\
Sdh3-F & TTTACGAAATGTTTGGAGACGC \\
Sdh3-R & GCTGGGAAGCAAGACGAGA \\
Actin-F & TATCGTTCTCGATTCTGGTGA \\
Actin-R & TCAAATCACGACCTGCAAGAT \\
\hline
\end{tabular}


3. Table S2. GO notes in cell component (CC), molecular function (MF) and biological process (BP).

\begin{tabular}{|c|c|c|c|c|c|}
\hline Category & GO ID & Term & Count & PValue & Up or Down \\
\hline \multirow{9}{*}{ GOTERM_BP } & GO:0009097 & isoleucine biosynthetic process & 3 & 0.005367738 & up \\
\hline & GO:0002181 & cytoplasmic translation & 10 & 0.002227167 & up \\
\hline & GO:0006412 & translation & 5 & 0.167527852 & up \\
\hline & GO:0042776 & mitochondrial ATP synthesis coupled proton transport & 3 & 0.013027726 & up \\
\hline & GO:0044710 & single-organism metabolic process & 9 & 0.03220119 & down \\
\hline & GO:0044281 & small molecule metabolic process & 7 & 0.03402374 & down \\
\hline & GO:0044711 & single-organism biosynthetic process & 6 & 0.061152645 & down \\
\hline & GO:0006541 & glutamine metabolic process & 2 & 0.07489116 & down \\
\hline & GO:0009084 & glutamine family amino acid biosynthetic process & 2 & 0.093697582 & down \\
\hline \multirow{4}{*}{ GOTERM_CC } & GO:0022625 & cytosolic large ribosomal subunit & 4 & 0.090090428 & up \\
\hline & GO:0016021 & integral component of membrane & 5 & 0.994147449 & up \\
\hline & GO:0044444 & cytoplasmic part & 21 & 0.015661627 & down \\
\hline & GO:0005737 & cytoplasm & 22 & 0.0245664 & down \\
\hline
\end{tabular}




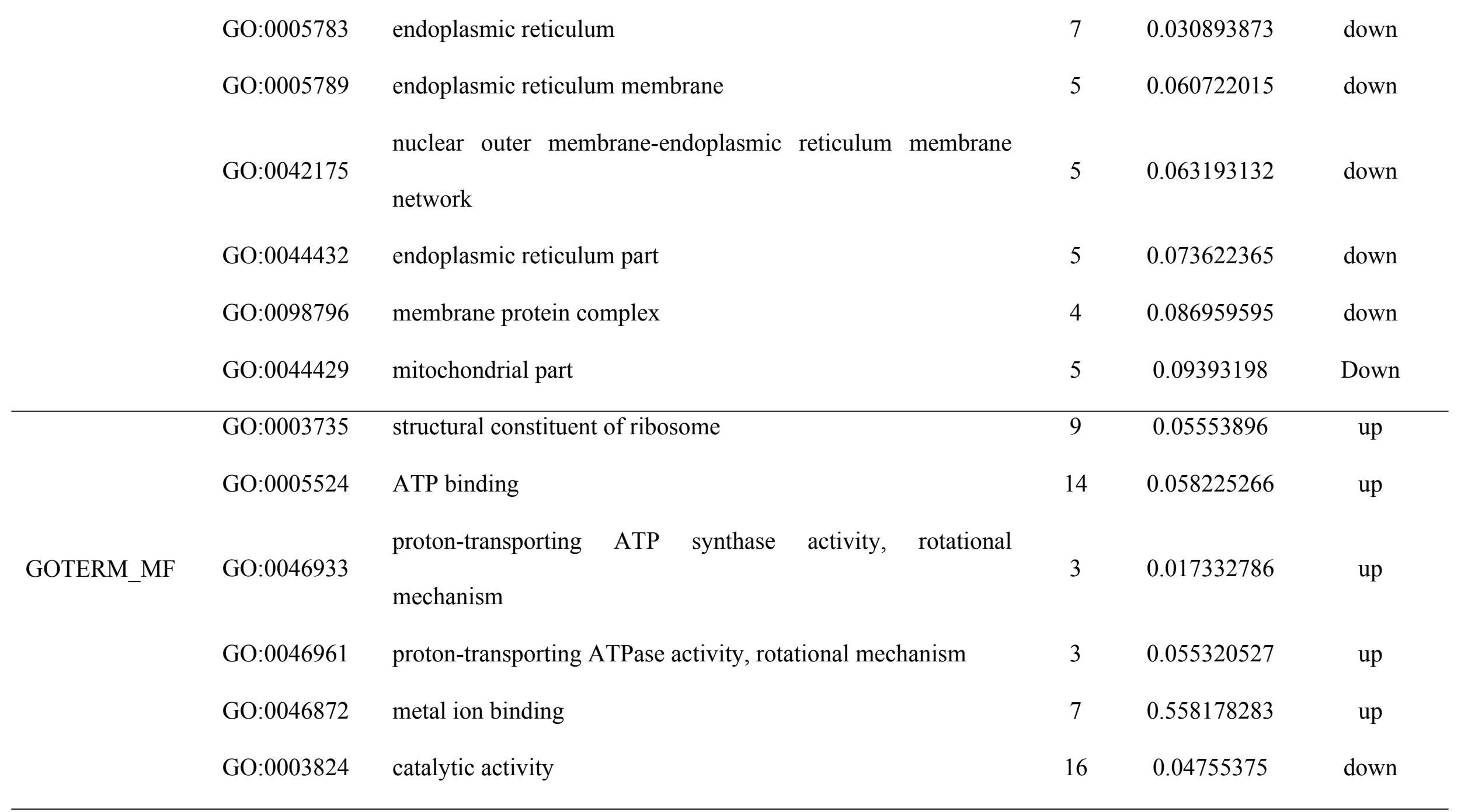


4. Table S3. Related pathways involve differential expression enzymes and genes in mitochondria.

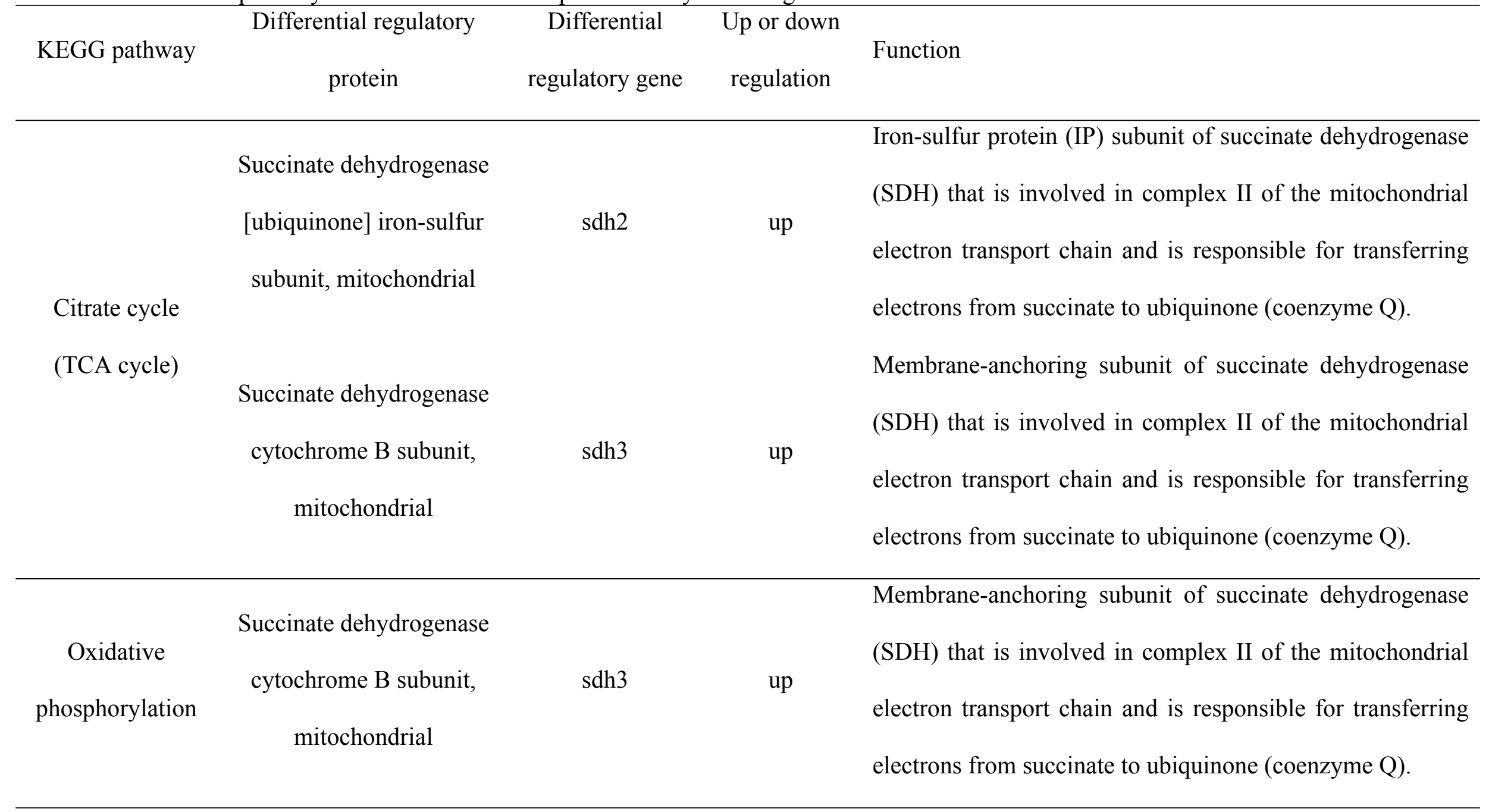




\section{Probable succinate \\ dehydrogenase}

[ubiquinone] flavoprotein

subunit, mitochondrial

Cytochrome c oxidase

subunit $6 \mathrm{~A}$, mitochondrial

$\operatorname{cox} 13$

up

ATP synthase subunit

alpha, mitochondrial
Flavoprotein (FP) subunit of succinate dehydrogenase

(SDH) that is involved in complex II of the mitochondrial electron transport chain and is responsible for transferring electrons from succinate to ubiquinone (coenzyme Q).

This protein is one of the nuclear-coded polypeptide chains of cytochrome $\mathrm{c}$ oxidase, the terminal oxidase in mitochondrial electron transport.

Mitochondrial membrane ATP synthase (F1F0 ATP synthase or Complex V) produces ATP from ADP in the presence of a proton gradient across the membrane which is generated by electron transport complexes of the respiratory chain. Subunits alpha and beta form the catalytic core in F1. Rotation of the central stalk against the surrounding alpha3beta3 subunits leads to hydrolysis of ATP in three separate catalytic sites on the beta subunits. 
Subunit alpha does not bear the catalytic high-affinity ATP-binding sites

Mitochondrial membrane ATP synthase (F1F0 ATP synthase or Complex V) produces ATP from ADP in the presence of a proton gradient across the membrane which is generated by electron transport complexes of the respiratory chain. Part of the complex F1 domain and the ATP synthase subunit gamma, mitochondrial central stalk which is part of the complex rotary element. The gamma subunit protrudes into the catalytic domain formed of alpha3beta3. Rotation of the central stalk against the surrounding alpha3beta3 subunits leads to hydrolysis of ATP in three separate catalytic sites on the beta subunits.

Mitochondrial membrane ATP synthase (F1F0 ATP synthase or Complex V) produces ATP from ADP in the presence of a proton gradient across the membrane which is 
generated by electron transport complexes of the respiratory chain. Part of the complex F0 domain. Minor subunit located with subunit a in the membrane.

Cytochrome c oxidase

polypeptide 5 ,

mitochondrial $\operatorname{cox} 5$

down
The transfer of electrons from cytochrome $\mathrm{c}$ to oxygen that occurs during oxidative phosphorylation, mediated by the multisubunit enzyme known as complex IV. 